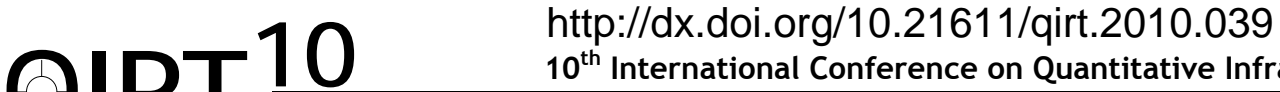 \\ $10^{\text {th }}$ International Conference on Quantitative InfraRed Thermography \\ July 27-30, 2010, Québec (Canada)
}

\section{Defect detection and characterisation in composite materials using active IR thermography coupled with SVD analysis and thermal quadrupole modeling}

\author{
by V. Feuillet* , L. Ibos*, M. Fois*, J. Dumoulin**, Y. Candau* \\ * Université Paris-Est, CERTES, 61 avenue du Général De Gaulle, 94010 Créteil Cedex, France, \\ vincent.feuillet@u-pec.fr \\ ** Université Paris-Est, LCPC, Route de Bouaye, BP 4129, 44341 Bouguenais Cedex, France
}

\begin{abstract}
In this study, a ctive infrared thermography is used to detect and characterize defects in carbon/epoxy composite plates. Defects are polymeric discs inserted between plies at different depths of the sample. The thermal excitation consists in a f inite time step us ing halogen lamps. The transient thermal modeling provides a one-dimensional analytical solution through thermal quadrupoles. Finally an inversion procedure is carried out to estimate modeling unknown parameters, especially the depth and thermal resistance of the defect.
\end{abstract}

\section{Introduction}

Non-destructive defect detection is a $\mathrm{m}$ ajor issue in the domain of composite materials for many application fields including aeronautics, civil engineering... Among standard techniques, active infrared thermography is a useful tool to detect material defects just after the process or in service. The thermal excitation can be of different nature.

In the flash method [1] the studied sample is i rradiated by a uni form he at pul se on on e side while the transient temperature either on the same side (front face experiment) or on the opposite side (rear face experiment) is recorded using an infrared camera. The main drawback of this well known-method is the compromise between flash intensity, exposition time, sample temperature increase and thermal wave penetration depth.

The s tep he ating method [2] is a nother widely used method in which the heating time is finite in order to better control the thermal wave penetration depth. This also permits the use of heat sources such as RF induction or halogen lamps where high peak power is often not available. To our knowledge this approach is used through front face observations.

In the present paper long pulse excitation was used coupled with front and rear faces infrared measurements. Two identification thermal models were developed based on quadrupole method [3]. One leads (using rear face data) to the characterization of thermophysical properties of the sound part of the material (composite plates). The second one (using front face data) leads to the identification of the depth and thermal resistance of a defect inserted in the material. Application of this approach was done over a set of 4 composite plates. An inverse procedure is carried out in two steps.

\section{Experimental setup}

\subsection{Sample characteristics}

Composite p lates of $21 \times 29.7 \mathrm{~cm}^{2}$ were m anufactured by Aircelle Le Havre ${ }^{\odot}$ (SAFRAN Group ${ }^{\odot}, \mathrm{F}$ rance). T hese carbon/epoxy composites have a fiber volume fraction of $\varphi=56 \%$ and a thickness of $2.35 \pm 0.07 \mathrm{~mm}$. Eight plies were laid up in a quasi-isotropic sequence $\left[0 \%+45^{\circ} /-45^{\circ} / 90^{\circ}\right]_{S}$ (figure 1) cured 1 hour at $180^{\circ} \mathrm{C}$ under a pressure of 7 bars and post-cured 4 hours at $190^{\circ} \mathrm{C}$ at atmospheric pressure. The composite pl ies consist of woven carbon fibers pre-impregnated with the epoxy resin. Carbon fibers are high resistance T300 fibers. The resin consists of two prepolymers TGDDM and TGpAP cross-linked with an aliphatic amine DDA. It contains an important proportion of toughening PES.

Disc defects of $1 \mathrm{~cm}$ diameter were inserted between plies in the centre of the composite plate at different depths (figure 1). In samples B02, B03 and B04, Teflon ${ }^{\odot}$ (PTFE) discs were inserted between plies 1 and 2 (B02), 2 and 3 (B03) and 4 and 5 (B04). For the B06 sample a disc of (PE, PTFE) copolymer thinner than the Teflon discs was inserted between plies 2 and 3 .

Thermal c onductivity $v$ alues gi ven by the m anufacturer ar e eq ual to $k_{r}=0.4 \mathrm{~W} \cdot \mathrm{m}^{-1} \cdot \mathrm{K}^{-1}$ for $\mathrm{t}$ he $\mathrm{r}$ esin a nd $\mathrm{o}$ $k_{f}=5 \mathrm{~W} \cdot \mathrm{m}^{-1} \cdot \mathrm{K}^{-1}$ for $\mathrm{c}$ arbon fibers in the transverse direction. The us e of a s eries model allows computing a $\mathrm{v}$ alue of $k=0.83 \mathrm{~W} \cdot \mathrm{m}^{-1} \cdot \mathrm{K}^{-1}$ for the composite plates thermal conductivity: 


$$
\frac{1}{k}=\frac{1-\varphi}{k_{r}}+\frac{\varphi}{k_{f}}
$$

The infrared emissivity of these composite plates was measured at $0.89 \pm 0.08$. Emissivity measurements were performed using a portable emissivity measurement device described in reference [4].

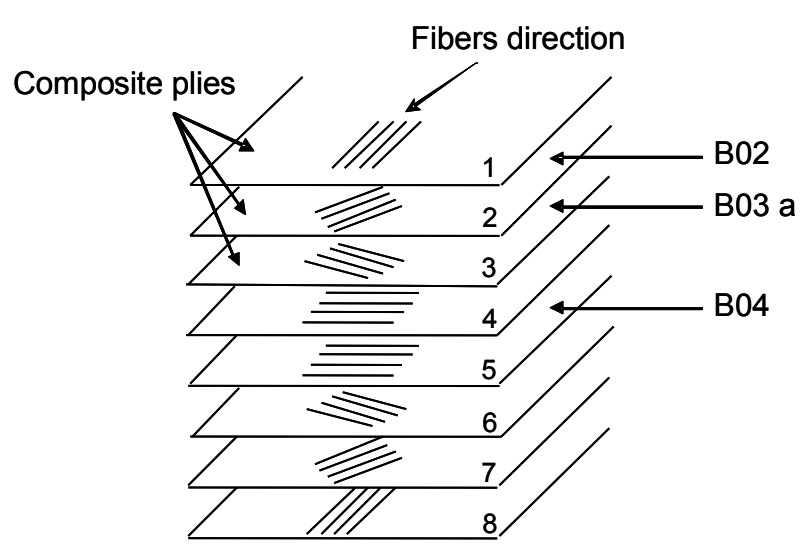

Fig. 1. Schematic representation of fibres orientation in composite samples and of defects position for each composite sample, namely B02, B03, B04, BO6.

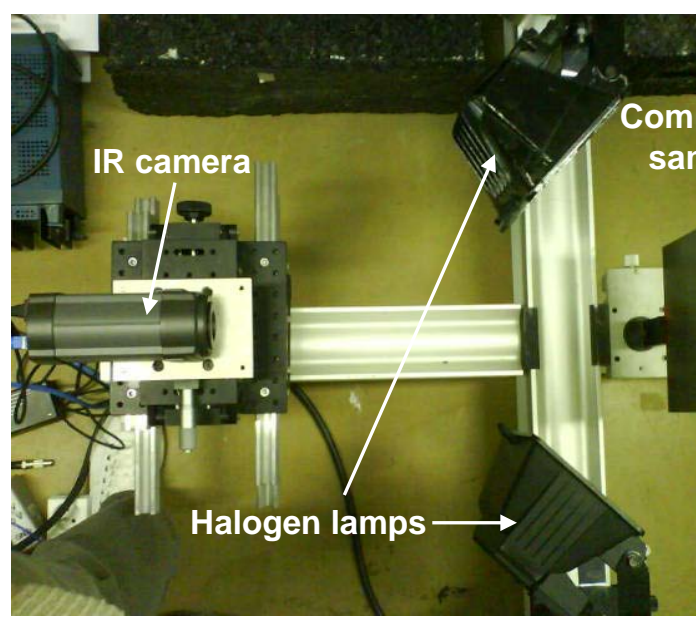

Fig. 2. Experimental set-up (front face measurements configuration).

\subsection{Laboratory test bench}

Test bench was developed in laboratory (figure 2). A FLIR ${ }^{\odot}$ A325G long wave $(7.5-13 \mu \mathrm{m})$ uncooled IRFPA camera was used to acquire thermal images during the heating and cooling phases. Temperature measurements were done on front and rear faces but not simultaneously. One side of the plates was heated with the step heating method using two halogen lamps of 500W each. It consisted in heating the sample for a given time length and in recording the increase and lowering of the temperature ev olution on the stimulated face or opposite face. Different heating periods (from 0.2 to $5 \mathrm{~s}$ ) were tested. Experimental protocol was controlled by a program developed under Labview ${ }^{\circ}$. Heat pul se d uration an d intensity were monitored. Two frequencies of temperature acquisition were used during experiments. The first one at $4 \mathrm{~Hz}$ consisted in storing full spatial resolution infrared image using real time averaging. The second one was set to $40 \mathrm{~Hz}$ and consisted in recording average thermograms over sound and defect areas.

\subsection{Experimental procedure}

First a rear face experiment on sound area allows to estimate different thermophysical parameters of the composite: the ratio between absorbed heat flux and thermal effusivity, the adimensional Biot number on front and rear faces and the diffusion constant t ime as sociated with the s ample. Thanks to these composite thermophysical c haracteristics a s econd estimation is carried out to characterize the defect. A front face experiment is used to estimate the defect depth and the ratio between defect and composite thermal resistances. Then the composite resistance was calculated from the thermophysical properties of the components.

\section{Heat transfer modeling}

In order to reduce the number of modeling unknown parameters, the direct thermal quadrupole model was rewritten by introducing several adimensional parameters in equations to improve the identification procedure. 


\subsection{Quadrupole modeling}

We consider a parallelepiped composite sample whose thickness is denoted $e_{0}$, thermal conductivity $k$ and thermal diffusivity a (figure 3 ). T he plate is s ubmitted to h eat e xchange both on $f$ ront an $\mathrm{d} r$ ear $\mathrm{f}$ aces ( global $\mathrm{t}$ hermal $\mathrm{t}$ ransfer coefficients $h$ ). One must distinguish a sound area with one layer of composite (figure 3(a)) from a defect area with two layers of composite separated by the defect (figure 3(b)). In this study, the defect thickness is known to be very small and the defect layer is modeled by a thermal resistance $R_{i}$.

(a)

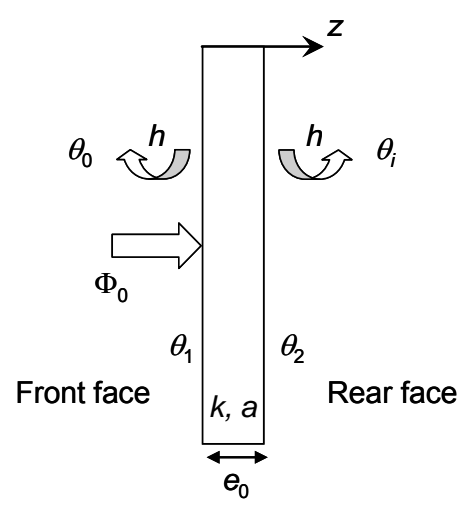

(b)

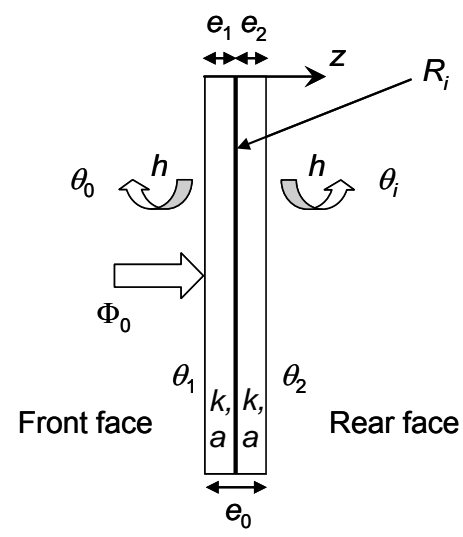

Fig. 3. Schematic view of the heat transfer modeling: (a) sound area, (b) defect area.

In the case of a uniform thermal excitation $\phi_{0}$ on the front face, heat transfer inside the sample is considered 1D in the $z$-direction and $t$ he diffusion in the plane of the composite plate is negl ected $s$ ince the thickness $e_{0}$ of the $s$ ample $(2.3 \pm 0.07 \mathrm{~mm})$ is very small compared to its lateral lengths $\left(21 \times 29.7 \mathrm{~cm}^{2}\right)$. The thermal quadrupole method based on integral transforms is us ed to solve the heat transfer problem corresponding to figure 3 [3]. A Laplace transform is applied to heat equation to link the temperature-heat flux density spectra $\theta_{1}$ and $\phi_{1}$ on the front face of the sample to the corresponding parameters $\theta_{2}$ and $\phi_{2}$ on the rear face, both on sound and defect areas:

$$
\begin{gathered}
{\left[\begin{array}{l}
\theta_{1} \\
\Phi_{1}
\end{array}\right]=\left[\begin{array}{ll}
A_{0} & B_{0} \\
C_{0} & D_{0}
\end{array}\right]\left[\begin{array}{cc}
1 & 1 / h \\
0 & 1
\end{array}\right]\left[\begin{array}{l}
\theta_{i} \\
\Phi_{i}
\end{array}\right]=\left[\begin{array}{ll}
A & B \\
C & D
\end{array}\right]\left[\begin{array}{l}
\theta_{i} \\
\Phi_{i}
\end{array}\right] \text { and }\left[\begin{array}{c}
\theta_{2} \\
\Phi_{2}
\end{array}\right]=\left[\begin{array}{cc}
1 & 1 / h \\
0 & 1
\end{array}\right]\left[\begin{array}{c}
\theta_{i} \\
\Phi_{i}
\end{array}\right] \text { sound area (2) }} \\
{\left[\begin{array}{l}
\theta_{1} \\
\Phi_{1}
\end{array}\right]=\left[\begin{array}{ll}
A_{1} & B_{1} \\
C_{1} & D_{1}
\end{array}\right]\left[\begin{array}{cc}
1 & R_{i} \\
0 & 1
\end{array}\right]\left[\begin{array}{ll}
A_{2} & B_{2} \\
C_{2} & D_{2}
\end{array}\right]\left[\begin{array}{cc}
1 & 1 / h \\
0 & 1
\end{array}\right]\left[\begin{array}{c}
\theta_{i} \\
\Phi_{i}
\end{array}\right]=\left[\begin{array}{ll}
A & B \\
C & D
\end{array}\right]\left[\begin{array}{c}
\theta_{i} \\
\Phi_{i}
\end{array}\right] \text { defect area (3) }}
\end{gathered}
$$

where the matrix transfer coefficients $A_{i}, B_{i}, C_{i}$ and $D_{i}(i=0,1,2)$ are associated to a given sample layer $i$ of thickness $e_{i}($ see figure 3) and equal to:

$$
A_{i}=D_{i}=\cosh \left(\sqrt{\tau_{i} p}\right) \quad B_{i}=\frac{\sinh \left(\sqrt{\tau_{i} p}\right)}{b \sqrt{p}} \quad C_{0}=b \sqrt{p} \sinh \left(\sqrt{\tau_{i} p}\right)
$$

with $p$ the Laplace variable, $\tau_{i}$ a diffusion time constant equal to $e_{i}^{2} / a$ and $b\left(=k / a^{1 / 2}\right)$ the thermal effusivity of the sample.

Eqs. (2) and (3) are solved analytically to obtain especially:

- the temperature spectrum on rear face for a sound area:

$$
\theta_{2}=\frac{\frac{\Phi_{0}}{b} \sqrt{\tau}}{\sqrt{\tau p} \times\left[\sinh (\sqrt{\tau p}) \times\left(\frac{B i^{2}}{\tau p}+1\right)+\cosh (\sqrt{\tau p}) \times\left(\frac{2 B i}{\sqrt{\tau p}}\right)\right]}
$$


where $B i=h e_{0} / \mathrm{k}$ is the Biot number on front and rear faces.

- $\quad$ the temperature spectrum on front face for a defect area:

with:

$$
\begin{gathered}
\theta_{1}=\frac{\frac{\Phi_{0}}{b} \sqrt{\tau}}{B i+\sqrt{\tau p} Y} \\
Y=\frac{[\Gamma \sqrt{\tau p} \times \sinh (\delta \sqrt{\tau p})+\cosh (\delta \sqrt{\tau p})] \times X+\sinh (\delta \sqrt{\tau p}) \times Z}{[\Gamma \sqrt{\tau p} \times \cosh (\delta \sqrt{\tau p})+\sinh (\delta \sqrt{\tau p})] \times X+\cosh (\delta \sqrt{\tau p}) \times Z} \\
X=\frac{B i}{\sqrt{\tau p}} \cosh ((1-\delta) \sqrt{\tau p})+\sinh ((1-\delta) \sqrt{\tau p}) \\
Z=\cosh ((1-\delta) \sqrt{\tau p})+\frac{B i}{\sqrt{\tau p}} \sinh ((1-\delta) \sqrt{\tau p})
\end{gathered}
$$

where:

- $\Gamma=R_{i} / R$ (with $R=e_{0} / \mathrm{k}$ ) is the ratio between the defect thermal resistance $R_{i}$ and the sample thermal resistance $R$. The defect thermal resistance $R_{i}$ is the sum of the disc thermal resistance and the thermal contact resistances.

- $\delta=e_{1} / e_{0}$ is the ratio between the defect depth and the sample thickness.

Finally an inverse Laplace transform is performed numerically in Eqs (5) and (6) to return to the time domain by using the de Hoog algorithm [5].

\subsection{Inverse procedure}

The parameters to estimate from temperature measurements and inverse procedure are $\Phi_{0} / b, B i, \tau, \delta$ and $\Gamma$. The last two parameters $\delta$ and $\Gamma$ allow to characterize the defect through its depth and thermal resistance.

Two experiment steps were used: one on rear face measurements for a sound area and another one on front face measurements for a def ect area. The rear face measurements give a better es timation of the composite thermophysical properties while front face measurements are well-suited to the defect characteristics estimation. The inverse problem is formulated in the least-squares sense and consists in finding the optimal solution that minimizes the functional:

$$
S=\sum_{j=1}^{J}\left[T_{\text {meas }, j}-T_{\text {estim }, j}(\beta)\right]^{2}
$$

where $T_{\text {meas }}$ are the measured temperatures, $T_{\text {estim }}$ are the es timated temperatures computed thanks to the he at transfer modeling presented in 3.1 by using $\beta$ vector constituted by the parameters to estimate, $J$ is the number of experimental data. $\beta$ vector is given as follows:

$$
\beta=\left[\Phi_{0} / b ; B i ; \tau\right] \text { in experiment type } 1 \text { and } \beta=\left[\Phi_{0} / b ; \delta ; \Gamma\right] \text { in experiment type } 2
$$

Minimization of $S$ is realized by using Levenberg-Marquardt algorithm [6] in order to estimate the components of $\beta$.

\section{Results}

\subsection{Laboratory experiments and contrast computations}

For the four plates, four step heating durations were studied $(0.5 \mathrm{~s}, 1 \mathrm{~s}, 2 \mathrm{~s}, 5 \mathrm{~s})$ both on front and rear faces. Simultaneously r ecordings of av erage thermal i mage s equences and thermograms over $s$ ound a nd d efect ar eas were realized at $4 \mathrm{~Hz}$. A cquisition of thermograms for identical thermal solicitation were realized at a hi gher frequency $(40 \mathrm{~Hz})$. Figure 4 shows absolute thermal contrast evolutions (temperature difference between a defect area and a sound area) for a given heating period (0.5s) applied on the front side. We recall that B02, B03 and B04 samples contain PTFE discs at 
different depths, B06 sample is the same as B03 with a thinner disc made of (PE, PTFE) copolymer. As expected, maximum value of thermal contrast is decreasing when defect depth increases. In the same way, the time corresponding to the maximum of $t$ hermal $c$ ontrast $i$ ncreases when $d$ efect depth i ncreases. $T$ he $m$ aximum of $t$ hermal $c$ ontrast and $t$ he corresponding time obtained for B 06 s ample are lower than those obtained for B 03 sample, whereas def ect depth is the same for both samples. This indicates that defect thermal resistance is lower in sample B06 than in sample B03.

Absolute contrast obtained for B04 sample using a heating duration of 0.5 s is very low. So, we present in figure 5 the evolution of absolute thermal contrasts for B04 sample using four different step heating durations varying from $0.5 \mathrm{~s}$ up to $5 \mathrm{~s}$. Heating duration higher than $2 \mathrm{~s}$ allows to obtain a maximum value of the thermal contrast greater than $0.5^{\circ} \mathrm{C}$ and thus seems more appropriate.

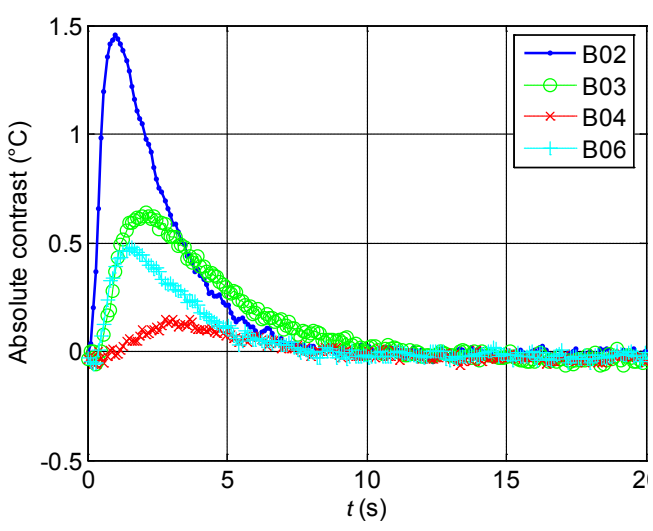

Fig. 4. Experimental absolute contrast evolutions for a heating period of $0.5 \mathrm{~s}$ on front side of the different samples.

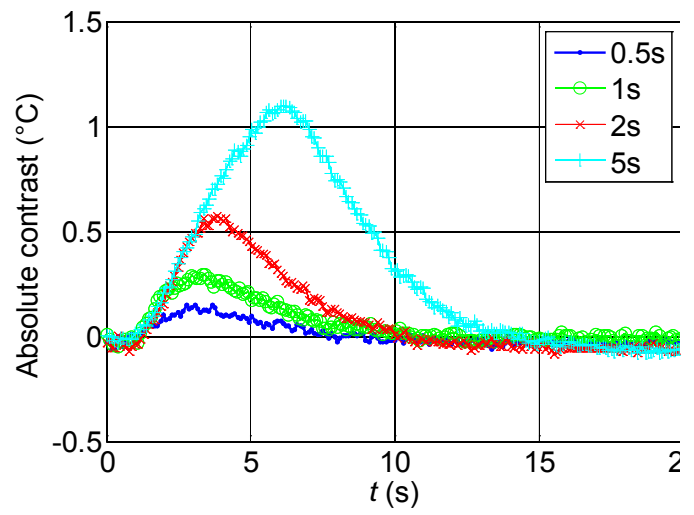

Fig. 5. Experimental absolute contrast evolutions for sample B04 and using different heating durations (0.5 to 5s).

\subsection{Analysis using singular value decomposition}

Singular value decomposition (SVD) is an interesting tool for the extraction of the spatial and temporal information from a thermographic matrix in a compact or simplified manner. The SVD of an $M x N$ matrix $\mathbf{A}(M>N)$ can be calculated as follows [7]:

$$
\mathbf{A}=\mathbf{U R V}^{T}
$$

where $\mathbf{U}$ is an $M x N$ orthogonal matrix, $\mathbf{R}$ being a diagonal $N x N$ matrix (with the singular values of $\mathbf{A}$ in the diagonal) and $\mathbf{V}^{T}$ is the transpose of an $N x N$ orthogonal matrix (characteristic time).

After $r$ earranging the thermograms for ev ery time as c olumns in $A$ and app lying the $S$ VD, the $c$ olumns of $U$ represent a set of orthogonal statistical modes known as empirical orthogonal functions (EOF) that describe spatial variations of data [8]. On the other hand, the principal components (PCs), which represent time variations, are arranged row-wise in matrix $\mathbf{V}^{T}$. The first EOF will represent the most characteristic variability of the data; the second EOF (denoted further as EOF2) will contain the second most important variability, and so on. Usually, original data can be adequately represented with only a few EOFs. SVD computations were performed using a sequence of 220 images acquired at a frequency of $4 \mathrm{~Hz}$. The image sequence starts at the beginning of the sample heating.

Figure 6(a) presents EOF2 maps obtained for the four composite samples using a heating duration of $0.5 \mathrm{~s}$. The presence of the defect is clearly seen, thus the method can be used to localize the position of one defect without any prior knowledge on its properties or position. We note that the amplitude in the center of the defect is greater for $\mathrm{BO} 2$ sample and decreases when defect depth increases. This can also be observed on the plots of principal component number 2 presented in figure 7. Principal component temporal evolution exhibits a peak whose amplitude and time position is correlated to the defect dept h a s obs erved in figure $4 \mathrm{f}$ or ab solute contrast. However, this kind of anal ysis r equires the us e of a hi gher sampling frequency to obtain accurate estimations of defect properties. Figure 6(b) presents EOF2 maps obtained for BO4 sample considering four different heating durations. It is clearly seen that the increase of heating duration from $0.5 \mathrm{~s}$ to $5 \mathrm{~s}$ allows obtaining def ect s ignature of increasing am plitudes and $t$ hus to r educe the i nfluence of m easurement noi se. The principle components as sociated temporal evolutions corresponding to these EOF maps are presented in figure 8 . We can observe that the peak amplitude is conserved whatever the heating duration, but the peak location is shifted towards longer times when heating duration is increased. Finally, the use of a very long heating duration (10s) shows an opposite effect on 
EOF2 map: a negative amplitude is obtained in the defect region. Moreover, the principal component behaviour shows also an opposite behaviour. This can be explained by the fact that the thermal manifestation of the defect starts in this particular case before the end of the heating duration. So, SVD may also bring information on the choice of experimental parameters, for instance indicate if the heating duration used is not too long.

(a)

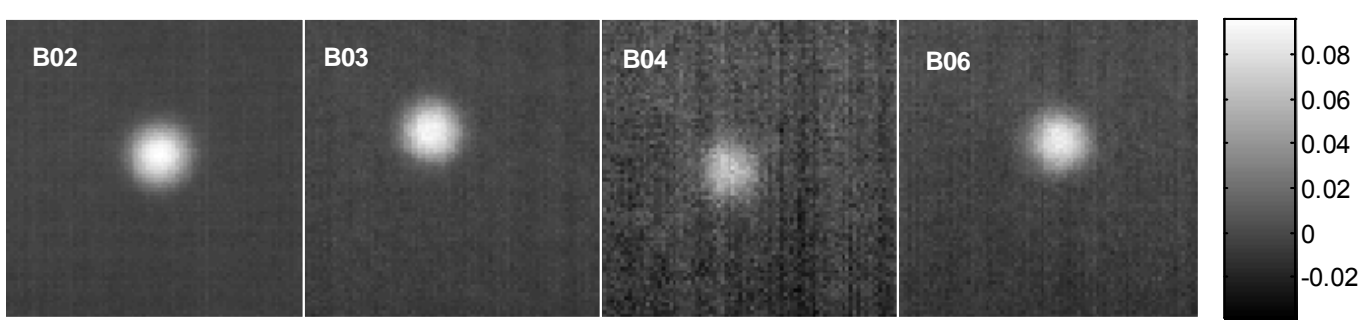

(b)

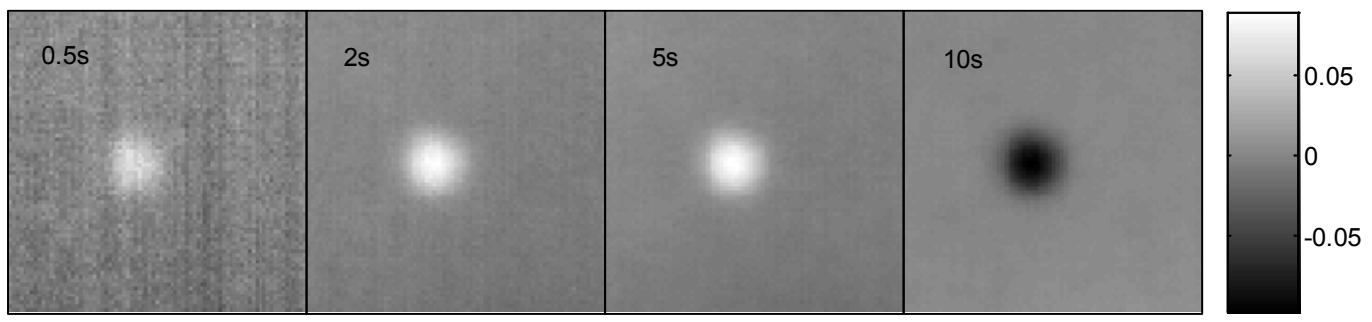

Fig. 6. Spatial EOF2 maps computed with experimental data: (a) front face experiments for a step heating time equal to 0.5 s with the four composite samples, (b) front face experiments with B04 sample for different heating durations.

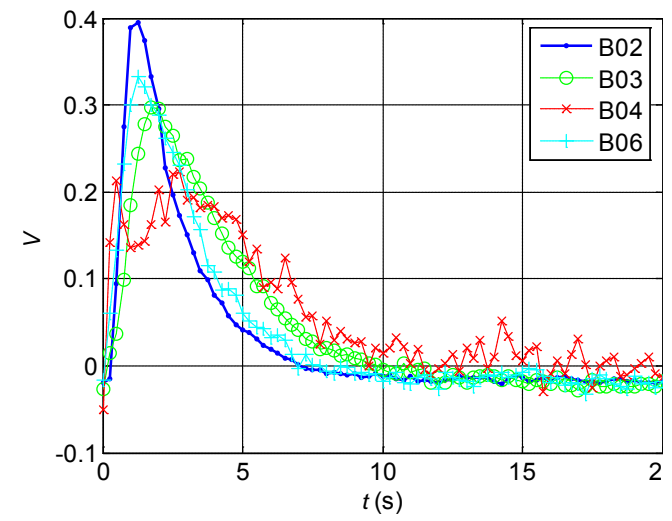

Fig. 7. Temporal evolutions of EOF2 Principal Component for a heating period of $0.5 \mathrm{~s}$ on front side of the different samples.

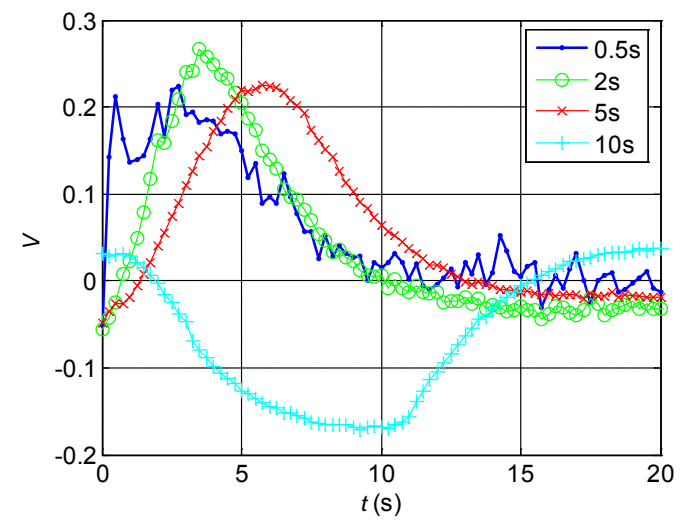

Fig. 8. Temporal evolutions of EOF2 Principal Component for sample B04 and using different heating durations (0.5 to 5s).

\subsection{Estimation results}

In this part the estimated parameters associated with each composite sample and experiment are computed. The inverse procedure was performed by using the SVD analysis to detect sound and defect areas.

First some estimation results concerning the B02 sample are presented in figure 9. Figures $9(\mathrm{a})$ and 9 (b) show that measured and estimated difference temperatures $\Delta T(t)=T(t)-T(t=0)$ are in good agreement for both rear face / sound area 
experiment (type 1) and front face / defect area experiment (type 2) after minimization of the functional in Eq. (8). Moreover reduced sensitivity coefficients $C_{i}$ are presented in Figures $9(\mathrm{c})$ and $9(\mathrm{~d})$. These coefficients are expressed by:

$$
C_{i}=\beta_{i} \frac{\delta T_{\text {estim }}}{\delta \beta_{i}}
$$

where $\beta_{i}$ are the p arameters to es timate (see E q. (9)), $\delta T_{\text {estim }}$ and $\delta \beta_{i}$ refer to v ery small v ariations r espectively of the estimated temperatures and the parameter $\beta_{i}$. These profiles indicate that parameters to es timate are not correlated since sensitivity curves present different shapes, what is suitable for an efficient identification. In addition we can observe that the sensitivity coefficient values associated with the Biot number are quite low along the experiment, so this parameter does not play an essential role on the temperature of the composite sample and on the inverse procedure.

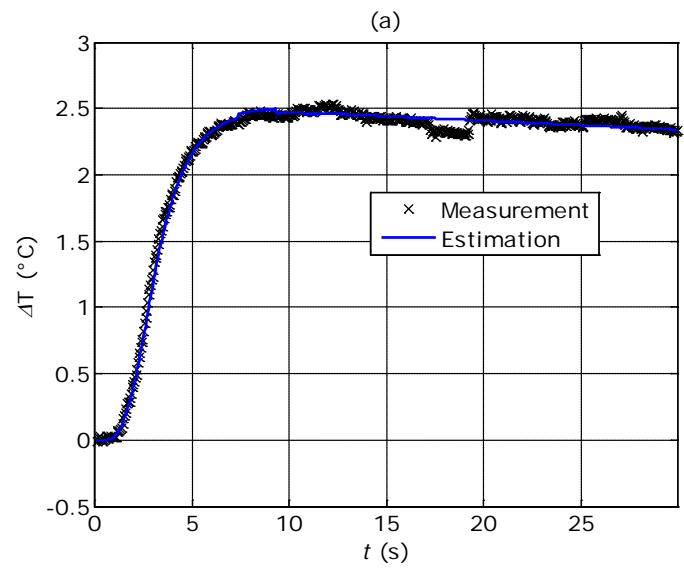

(c)

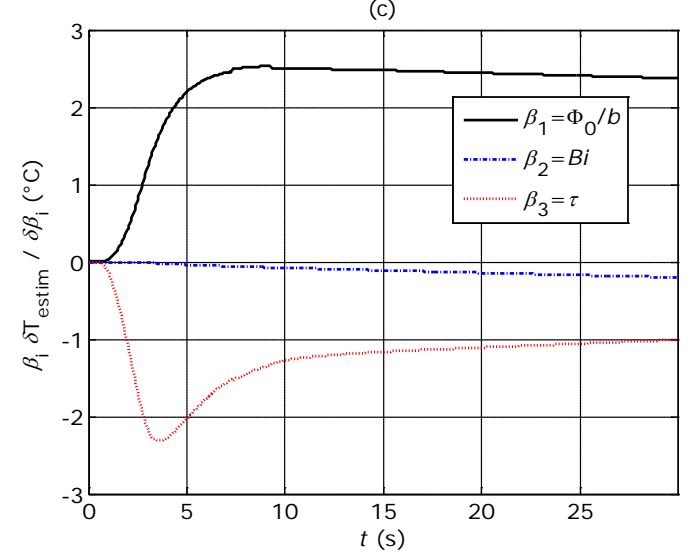

(b)

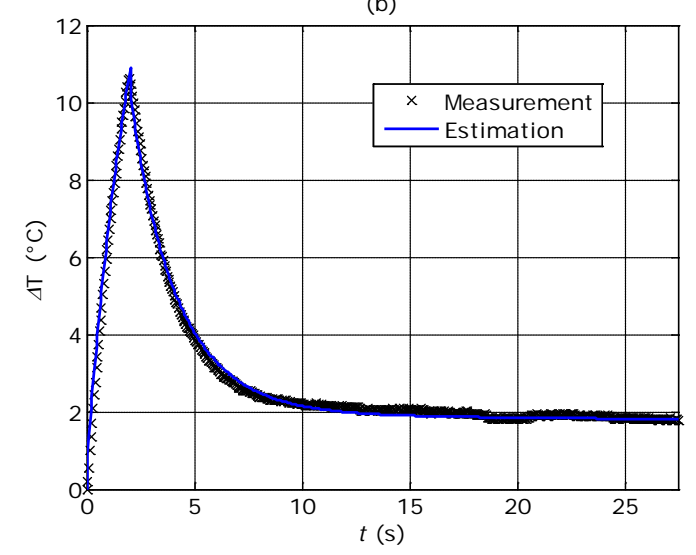

(d)

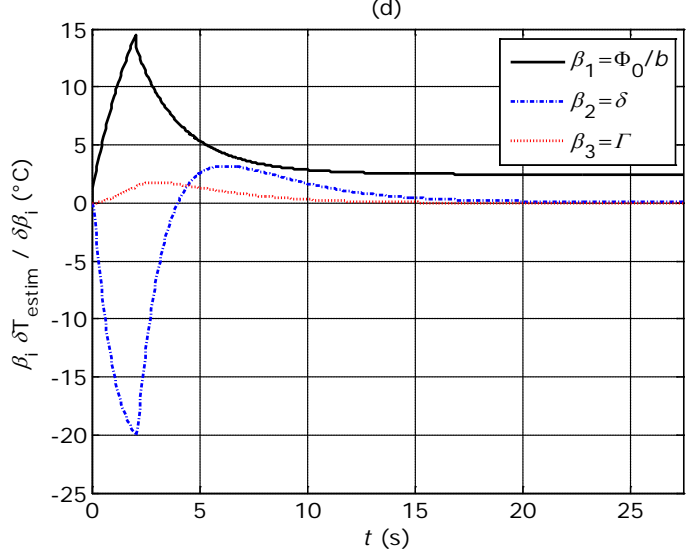

Fig. 9. Estimation results for the sample B02: (a) and (b) measured and estimated temperatures for experiments type 1 and 2, (c) and (d) reduced sensitivity coefficients for experiments type 1 and 2.

Table 1 summarizes estimation results obtained for the different samples and experiments.

All experiments t ype 1 ( rear f ace an $d$ s ound ar ea) lead to qui te s imilar es timated parameters, e specially t he diffusion constant time which is normally independent of the experiment type.

These previous es timated $p$ arameters were as sumed to be $k$ nown in experiments type 2 ( front face and defect area), excepted for the ratio $\Phi_{0} / b$ which is closely linked to the experimental conditions.

The different defect depths $e_{1}$ are deduced from the values of the adimensional parameter $\delta$. The theoretical defect depth $e_{t h}$ for each sample is known and can be compared to estimated values. Table 1 shows a satisfactory accuracy of the estimated defect depth.

Defect thermal resistance $R_{i}$ has also been estimated for each sample and was deduced from the values of the adimensional parameter $\Gamma$. For a step heating duration of $2 \mathrm{~s}$, estimated values for B02 and B03 samples were close whereas estimated defect thermal resistance for B04 sample was slightly lower. However the defect characteristics in samples B02, B03 and B04 should be the same. On the other hand, the increase of the step heating duration to 5s for B04 sample allowed to estimate a value equivalent to the previous ones. The more important defect depth in B04 sample required a higher step 
heating duration to increase the thermal wave penetration depth. Finally, as shown by studying the thermal contrast in Figure 4, we observed that (PE, PTFE) copolymer disc thermal resistance associated with B06 sample is lower than PTFE thermal resistance.

\begin{tabular}{|c|c|c|c|c|c|c|c|}
\hline \multirow[b]{2}{*}{$\begin{array}{c}\text { Composite } \\
\text { sample }\end{array}$} & \multirow[b]{2}{*}{$\begin{array}{c}\text { Experiment } \\
\text { type }\end{array}$} & \multirow[b]{2}{*}{$\begin{array}{c}\text { Heating } \\
\text { duration (s) }\end{array}$} & \multicolumn{5}{|c|}{ Estimated parameters } \\
\hline & & & $\phi_{0} / b\left(\mathrm{~K} . \mathrm{s}^{-1 / 2}\right)$ & $B i$ & $\tau(\mathrm{s})$ & $\frac{e_{t h}(\mathrm{~mm})}{e_{1}(\mathrm{~mm})}$ & $R_{i}\left(\mathrm{~K} \cdot \mathrm{m}^{2} \cdot \mathrm{W}^{-1}\right)$ \\
\hline \multirow[b]{2}{*}{ B02 } & RF & 2 & $4.93 \pm 0.02$ & $\mathbf{0 . 0 2 1 \pm 0 . 0 0 1}$ & $14.49 \pm 0.07$ & & \\
\hline & FF & 2 & $4.00 \pm 0.02$ & & & $\begin{array}{c}0.30 \\
0.33 \pm 0.01\end{array}$ & $\begin{array}{c}\text { 1.99E-03 } \\
\pm 0.03 E-03\end{array}$ \\
\hline \multirow[b]{2}{*}{ B03 } & RF & 2 & $4.68 \pm 0.03$ & $\mathbf{0 . 0 2 2} \pm 0.001$ & $14.78 \pm 0.11$ & & \\
\hline & FF & 2 & $4.72 \pm 0.02$ & & & $\begin{array}{c}0.57 \\
0.62 \pm 0.01\end{array}$ & $\begin{array}{c}\text { 2.09E-03 } \\
\pm 0.04 \mathrm{E}-03\end{array}$ \\
\hline \multirow{3}{*}{ B04 } & RF & 2 & $4.81 \pm 0.04$ & $\mathbf{0 . 0 1 9} \pm 0.002$ & $15.28 \pm 0.13$ & & \\
\hline & $\mathrm{FF}$ & 2 & $4.34 \pm 0.02$ & & & $\frac{1.15}{1.25 \pm 0.05}$ & $\begin{array}{c}\text { 1.30E-03 } \\
\pm 0.15 \mathrm{E}-03\end{array}$ \\
\hline & $\mathrm{FF}$ & 5 & $4.25 \pm 0.02$ & & & 1.15 & $\begin{array}{c}\text { 2.22E-03 } \\
\pm 0.15 E-03\end{array}$ \\
\hline \multirow[b]{2}{*}{ B06 } & RF & 2 & $4.16 \pm 0.02$ & $0.018 \pm 0.002$ & $11.92 \pm 0.21$ & & \\
\hline & FF & 2 & $4.02 \pm 0.01$ & & & $\begin{array}{c}0.57 \\
0.64 \pm 0.01\end{array}$ & $\begin{array}{c}1.59 E-03 \\
\pm 0.03 E-03\end{array}$ \\
\hline
\end{tabular}

Table 1. Estimation results (RF=rear face/sound area experiment, $F F=$ front face/defect area experiment).

\section{Conclusions}

Active IR thermography coupled with SVD analysis and thermal quadrupole modeling allowed us to characterize different defects in carbon/epoxy composite samples. The us e of s everal a dimensional parameters within the quadrupole modeling made possible to estimate both defect depth and thermal resistance. It appeared that SVD analysis is a powerful tool to differentiate a s ound area from a d efect ar ea and that the s tep he ating d uration plays a $n$ important $r$ ole on the estimation performance. Then the combination of front and rear face temperature measurements with an inverse procedure lead to estimate precisely the defect depths. In addition we could compare the thermal resistance of two different types of polymeric defects (PTFE and (PE, PTFE) copolymer discs).

\section{ACKNOWLEDGMENTS}

The authors acknowledge Prof. Hugues Duflo (LOMC, Le Havre University) who provided the composite samples.

\section{REFERENCES}

[1] Parker W J., "Flash Method of Determining Thermal Diffusivity, Heat Capacity and Thermal Conductivity", Journal Appl. Phys., 32, 1679-1684, 1961.

[2] Osiander R., Spicer J. W. M., "Time-resolved infrared radiometry with step heating. A review", Revue Générale de Thermique, 37, 650-692, 1998.

[3] Maillet D., André S., Batsale J. C., Degiovanni A. and Moyne C., "Thermal Quadrupoles", Wiley, Chichester, 2000.

[4] Ibos L., Marchetti M., Boudenne A., Datcu S., Livet J., Candau Y., "Infrared emissivity measurement device: Principle and applications", Measurement Science and Technology, 17, 2950-2956, 2006.

[5] de Hoog F. R., Knight J. H. and Stokes A. N., "An improved method for numerical inversion of Laplace transforms", SIAM Journal, 3, 357-366, 1982.

[6] Marquardt D. W., "An algorithm for the least squares estimation of non linear parameters", SIAM Journal, 1,431-441, 1963.

[7] Rajic N., "Principal component thermography for flaw contrast enhancement and flaw depth characterization in composite structures", Composite Structures, vol 58, pp 521-528, 2002.

[8] Dumoulin J., Ibos L., Ibarra-Castanedo C., Mazioud A, Marchetti M., Maldague X. and Bendada A., "Active infrared thermography applied to non emergent defects detection on asphaltic pavement samples: experiments and numerical simulations", Proceedings of 10th international workshop on advanced infrared technology and applications (AITA'09), Florence (Italy), 8-11 september 2009. 